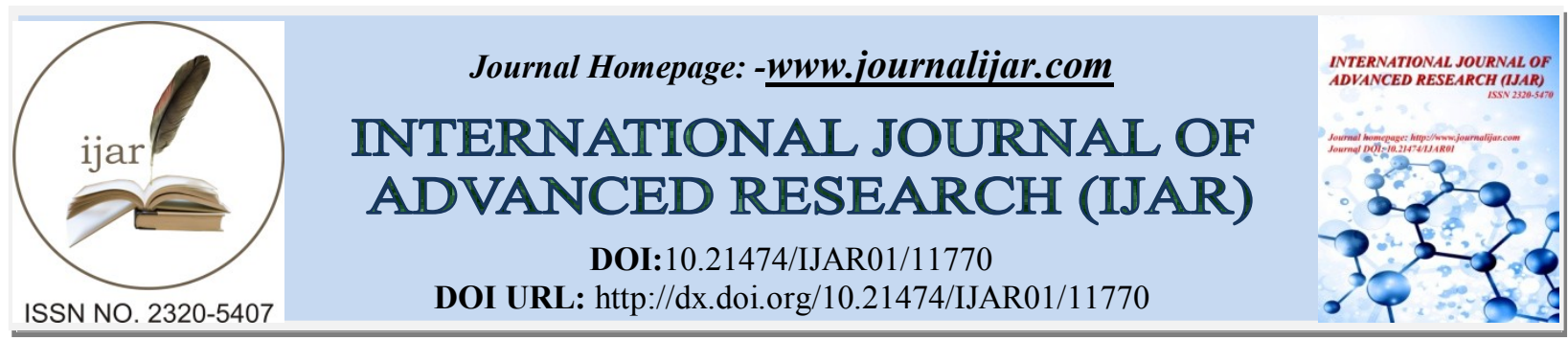

RESEARCH ARTICLE

\title{
IMPACT DES EFFLUENTS DES FOSSES SEPTIQUES SUR LA QUALITE DES EAUX SOUTERRAINES DES NAPPES SUPERFICIELLES DANS LA VILLE DE THIES, SENEGAL
}

\author{
Mouhamadou Masseck Fall ${ }^{1}$, Khadime Dieng ${ }^{1}$, Mamadou Lamine Lo ${ }^{1}$, Capitaine Falilou Sarr $^{2}$ and El. Hadji \\ Bamba Diaw ${ }^{1}$ \\ 1. Laboratoire des Sciences et Techniques de l'Eau et de l'Environnement (LaSTEE), Ecole Polytechnique de de \\ Thiès BP A10 Thiès, Sénégal. \\ 2. Hygiéniste/Environnementaliste, chef Service Régional de l'Hygiène de Diourbel, Sénégal
}

\section{Manuscript Info}

Manuscript History

Received: 20 July 2020

Final Accepted: 24 August 2020

Published: September 2020

Key words:-

Pollution, Eaux Souterraines, Fosses

Septiques, Puits, Thiès

\section{Abstract}

La forte tendance des populations citadines du Sénégal en général, et de la ville de Thiès en particulier, à recourir au fonçage de puits domestiques pour leur approvisionnement en eau potable les expose à des dangers d'infections dues à des risques de pollution des eaux souterraines d'origines chimiques, accidentelles, domestiques et bactériologiques. Ces polluants qui ont pour origine la surface du sol du fait de la proximité des puits avec les ouvrages d'assainissement autonomes comme les fosses septiques, peuvent atteindre la nappe phréatique suivant un mécanisme de transfert et d'évolution à travers les couches sus-jacentes de la nappe phréatique. Dans cette étude nous avons procédé par le prélèvement d'échantillons d'eaux au niveau treize (13) puits qui captent la nappe peu profonde et disséminés dans les trois (03) communes d'arrondissement de la ville de Thiès. Les analyses physico-chimiques et microbiologiques effectuées sur les ces échantillons, montrent une pollution sous forme de phosphates, de nitrates, d'une minéralisation très élevée et de par les coliformes fécaux, attestant ainsi leur contamination par les déchets humains et animaux issus des fosses septiques.

Copy Right, IJAR, 2020,. All rights reserved.

\section{Introduction:-}

A l'instar des pays en développement de façon générale et au Sénégal en particulier, la majeure partie des populations des zones urbaines et périurbaines ont encore recours aux sources d'approvisionnement traditionnelles en eau potable comme les puits qui exploitent la nappe phréatique. Par contre l'assainissement des eaux usées, facteur très important pour un équilibre environnemental parfait, fait souvent défaut. Le branchement à l'égout public facilite une bonne gestion des eaux usées domestiques et constitue une très bonne solution face à la pollution des nappes souterraines par les matières fécales d'origine humaine. Cependant les localités qui ne sont pas branchés à l'égout public utilisent des fosses septiques ne respectant pas les normes d'implantation et la technologie de construction.

Ainsi, la mauvaise qualité physicochimique et bactériologique de ces eaux de la nappe phréatique dans leur grande majorité est avérée, et laisse présager une pollution bactériologique et physicochimique de ces ressources due aux 
mauvais systèmes d'assainissement de façon générale et à leur proximité des fosses septiques en particulier. La prolifération des décharges incontrôlées parallèlement à l'urbanisation galopante vers les zones périurbaines a tendance à favoriser la bidonvilisation et la précarité des conditions de vie et de subsistance des populations. Et face à la cherté des factures d'eau, ces populations ont souvent recours, au fonçage de puits domestiques dans des parcelles de terrains très réduites, ou aux puits traditionnels, généralement impropres à la consommation. Ces eaux souterraines de puits peu profonds sont contaminées par les déchets humains et animaux, très solubles dans l'eau et constituent de nos jours la principale cause de la pollution des eaux souterraines [1].

Ce phénomène est très répandu au Sénégal et dans la ville de Thiès en particulier. Ainsi le présent article a pour objectif d'étudier, par le biais d'analyses physicochimique et bactériologiques, l'impact des fosses septiques la qualité des eaux de la nappe phréatique de plus en plus très prisées par les populations.

\section{Materiels Et Methodes:-}

\section{Description De La Zones D'étude}

Notre zone d'étude, la ville de Thiès st le chef-lieu de la région éponyme, deuxième ville du Sénégal après Dakar dont elle est située à $70 \mathrm{Km}$ à l'est. Elle couvre une superficie de $6600 \mathrm{Km}^{2}$ pour une population de d'environ 350 000 habitants en 2013. Son découpage administratif la subdivise en trois Communes d'Arrondissement (CA), ThièsNord, Thiès-Est et Thiès-Ouest.

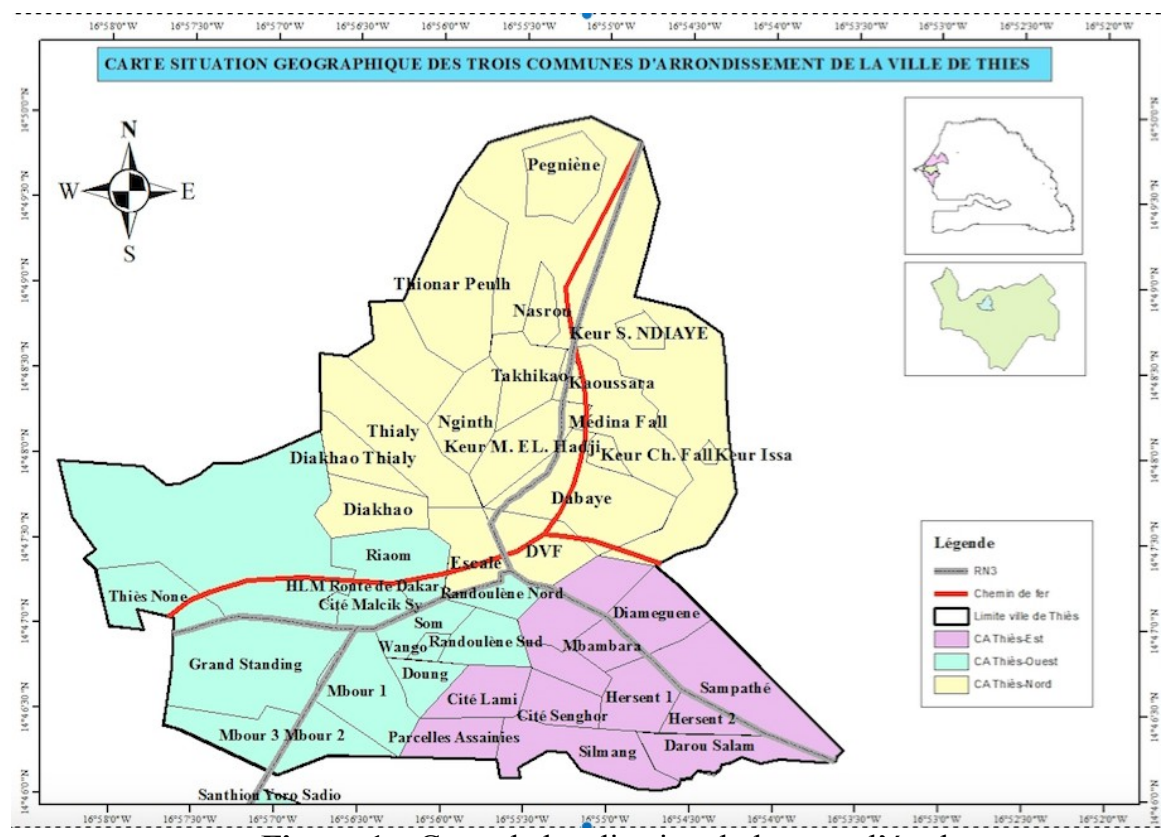

Figure 1:- Carte de localisation de la zone d'étude.

Thiès est limitée au Nord par la communauté rurale de Fandène, au Sud par La communauté rurale de Noto, à l'Est par les villages Keur Yoro Sadio et Baniaye, à l'Ouest par les villages Sène Wolof et Ngomène.

Du point de vu climatologique La zone est située en grande parties dans la zone climatique sahélo-soudanienne. Son régime climatique est déterminé comme dans toute l'Afrique de l'Ouest par le régime des précipitations. Elle est soumise à l'influence des trois vents : l'alizé, l'harmattan, vent chaud et sec et la mousson. Ainsi, la ville de Thiès possède une température moyenne de $26,7^{\circ} \mathrm{C}$, la pluviométrie moyenne annuelle est d'environ $450 \mathrm{~mm}$. Les vents viennent des directions Nord et Nord-Est, et de direction Ouest avec une vitesse moyenne de $4,15 \mathrm{~m} / \mathrm{s}$. L'humidité relative maximale notée dans la ville de Thiès est de $79 \%$. La pédologie de la ville de Thiès est caractérisée en majorité de $78 \%$ de sols diors. La topographie montre un relief qui plat en majorité, reposant sur des marnes et des calcaires. Le contexte hydrogéologique est marqué par la présence de nappes superficielles sur toute l'étendue de la ville de Thiès avec une profondeur moyenne de $13 \mathrm{~m}$. Cette présence de nappe phréatique est liée à la pluviométrie et à la géologie. Ainsi, plusieurs puits d'environ 12 à $13 \mathrm{~m}$ de profondeur en moyenne captant ces nappes ont été implantés par les populations pour satisfaire leur besoin en eau. 


\section{Méthodologie:-}

La méthodologie mise en œuvre a combiné deux approches principales et s'est appuyée sur une démarche prospective dans le but d'aboutir à une étude représentative de la zone d'étude.

Le choix des puits est basé sur leur exploitabilité à des fins domestiques, mais surtout sur le degré d'accès au réseau d'assainissement collectif dans la zone, ce qui impose aux populations exploitantes le recours au système d'assainissement autonome en fosses septiques.

A cet effet, deux campagnes de prélèvement d'échantillons d'eau de puits ont été effectuées dans la période du 05 juillet 2018 au 20 juin 2019. Le phasage suivant a été adopté et s'articule autour de trois axes.

\section{La phase prospective}

Elle a permis d'identifier et de choisir les ouvrages sur la base de leur fréquentation quotidienne et leur position géographique afin d'obtenir bon un maillage de la commune, en privilégiant les quartiers non encore desservis par le réseau d'égouts collectifs et qui utilisent comme système d'assainissement des fosses septiques.

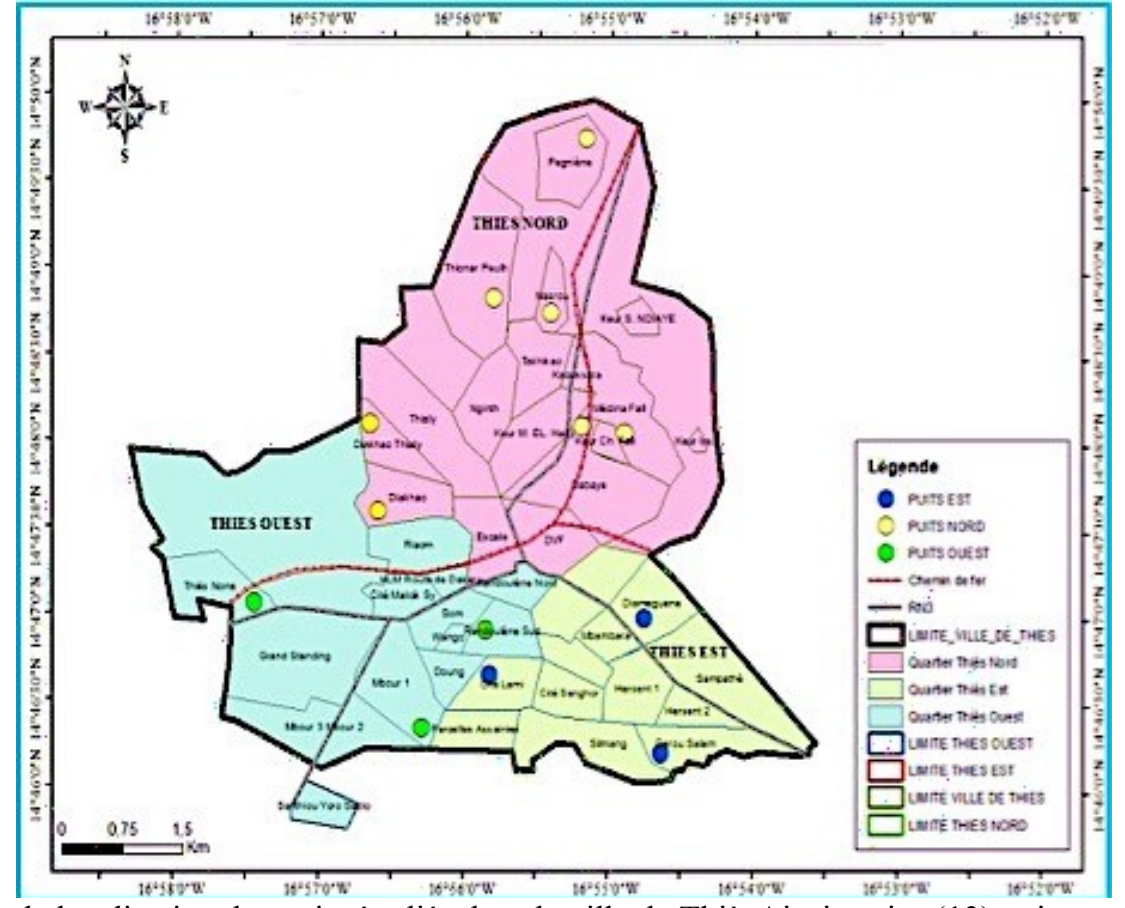

Figure 2:- Carte de localisation des puits étudiés dans la ville de ThièsAinsi, treize (13) puits ont été sélectionnés faisant l'objet de cette présente campagne d'échantillonnage.

\section{La phase préparatoire et d'échantillonnage}

Dans chaque puits nous avons effectué deux prélèvements de $600 \mathrm{ml}$ dont l'un est destiné aux analyses physicochimiques et l'autre à l'analyse microbiologiques. Les appareils de mesures in-situ et flacons de substances stabilisantes pour les analyses physicochimiques proviennent du laboratoire d'assainissement de l'Ecole Polytechnique de Thiès (EPT), et les contenants stérilisés destinés aux analyses microbiologiques sont fournis par le laboratoire de l'Office National d'Assainissement du Sénégal (ONAS). Les échantillons d'eau prélevés sont étiquetés, conditionnés et acheminés dans les quatre heures au laboratoire de l'EPT pour la caractérisation des paramètres physicochimiques, et dans les douze heures aux laboratoires de l'ONAS pour les analyses microbiologiques.

Pour mener à bien cette présente étude, nous nous sommes servis de deux pH-mètres de marques Consort C5010 et HI83141, un Conductimètre HI8733, des contenants appropriés et un lot de matériels d'échantillonnage de terrain. Au laboratoire, un Spectromètre AL800, un Microscope binoculaire, Stérilisateur à rayon UV, un Thermo-réacteur AL125, un Autoclave, une Balance électronique et une Pompe à vide entre autres matériels ont été mis en contribution. 


\section{Résultats et Discussions:-}

Les résultats d'analyses sont présentés en fonction des seuils de pollution de leurs concentrations en ions et en micro-organismes. Les paramètres physicochimiques sont représentés dans des graphiques avec les valeurs guides des eaux de consommation définies par l'organisation mondiale pour la santé (OMS) [2], les Directives du Conseil des Communautés Européennes (DCCE) et la Norme Française (NF) [3].

\section{LE PH}

Le $\mathrm{pH}$ conditionne les équilibres physico-chimiques de l'eau. Il est dit acide dans les eaux des aquifères sableuses ou granitiques lorsque sa valeur est inférieure à 7 , et alcaline ou basique dans les eaux des aquifères calcaires quand sa valeur est supérieure à 7 .

Près de la moitié des puits échantillonnés dans les trois CA de Thiès ne respecte pas les normes standards de l'OMS en matière de $\mathrm{pH}$ (compris entre 6,5 et 9,5).

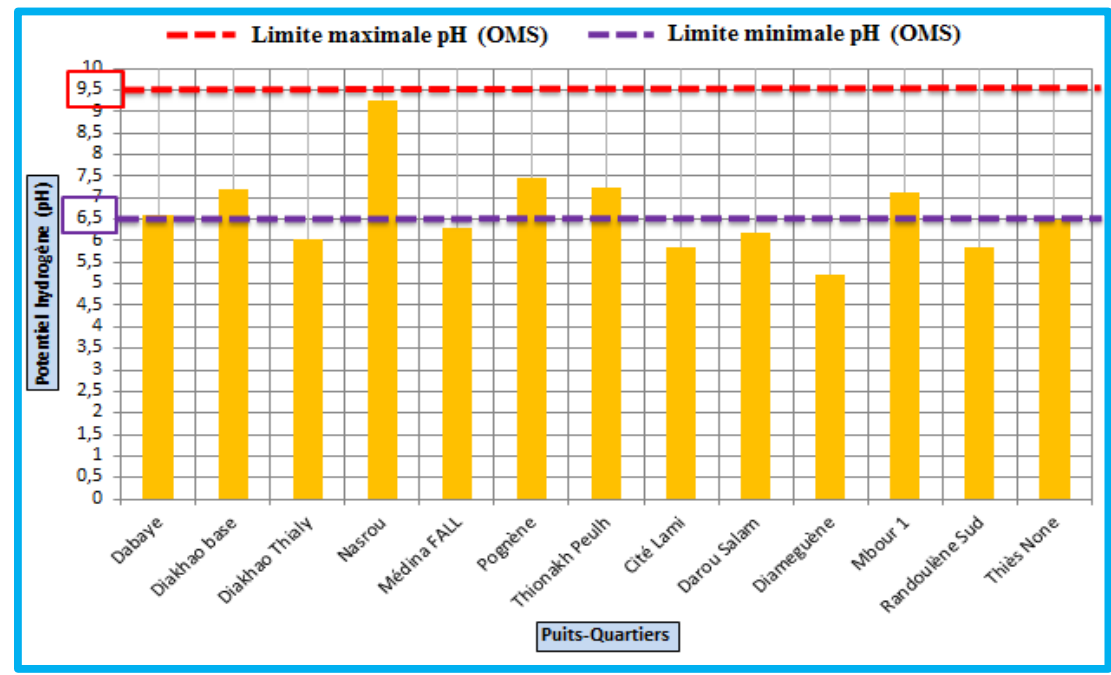

Figure 3:- Variation du pH dans les puits par rapport à la norme OMS.

L'eau du puits de Nasrou a le $\mathrm{pH}$ le plus élevé des ouvrages visités $(9,25)$; suivi de celle de Pognène $(7,47)$, alcalin situées dans une zone aquifère caractérisé par la présence de calcaires [4]. C'est le cas dans la CA de Thiès-Nord à Pognène, Thionakh Peulh et Diakhao base.

Les eaux provenant des terrains pauvres en calcaires ou siliceux ont un $\mathrm{pH}$ voisin de 7 et quelquefois un peu inférieur comme à Dabaye et Médina FALL qui sont respectivement de 6,6 et 7,25.

Les puits de Diamaguène, Cité Lamy, Randoulène sud et Diakhao Thialy dont les eaux ont des $\mathrm{pH}$ qui varient entre 5,23 et 6,03 sont plutôt acides, captant des aquifères sablo-graveleux [5].

Les puits dont les eaux ont des valeurs de $\mathrm{pH}$ respectent la norme du guide de l'OMS sont tous situés dans la commune d'arrondissement de Thiès Nord (Dabaye, Diakhao-Base, Nasrou, Pognène, Thionakh Peulh) à l'exception de celui de Mbour 1 situé dans la CA Thiès Est.

Les $\mathrm{pH}$ en deçà de la valeur minimale de l'OMS sont obtenus dans les eaux de puits de Diakhao-Thialy, MédinaFall dans la CA de Thiès Nord, Cité-Lami, Darou-Salam, Diamaguène dans la CA de Thiès-Est et Randoulène-Sud dans la CA de Thiès-Ouest. Ils représentent $46 \%$ de l'Échantillon. 


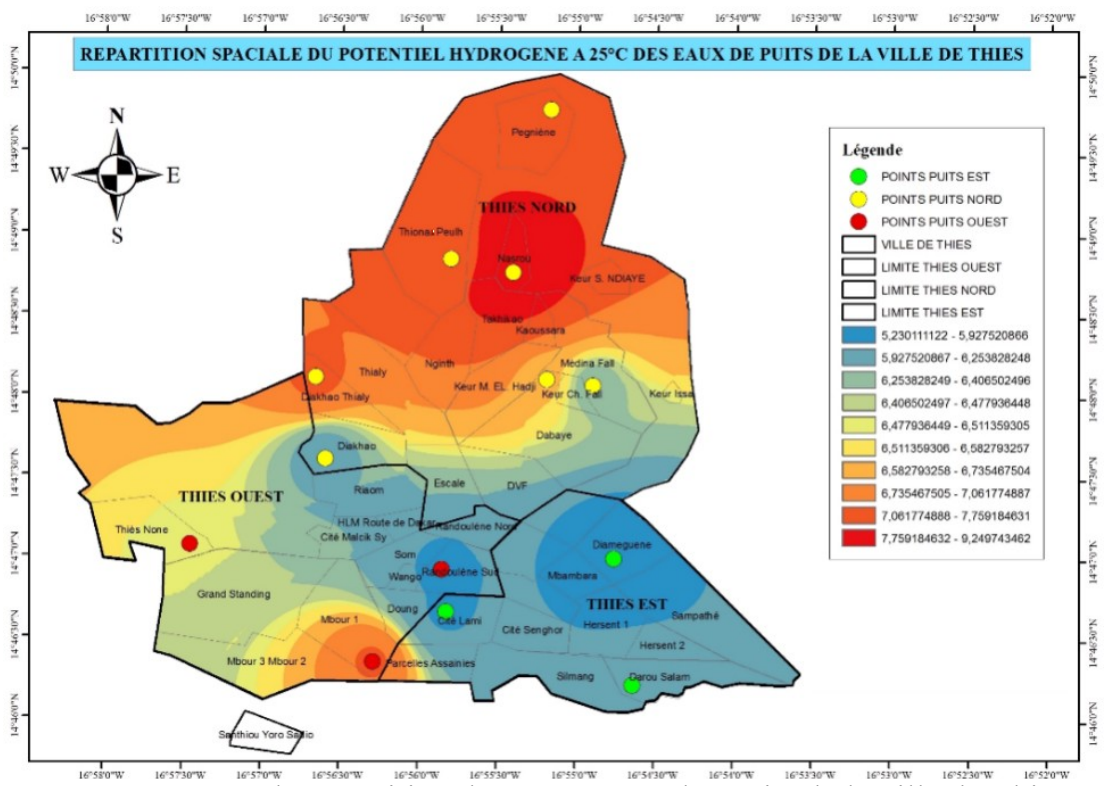

Figure 4:- Carte de répartition du $\mathrm{pH}$ à travers les puits de la ville de Thiès.

\section{La Conductivite}

Globalement, la conductivité électrique $(\mathrm{CE})$ est supérieure au seuil maximal de la norme $\mathrm{OMS}(400 \mu \mathrm{S} / \mathrm{cm})$ à l'exception du puits de Diakhao-Thialy, engendrant une minéralisation des eaux de puits allant d'importante à élevée dans la presque totalité des trois CA de Thiès [6]. Cette conductivité électrique varie entre 305 et $2630 \mu \mathrm{S} / \mathrm{cm}$ dans les puits de la CA de Thiès-Nord, entre 730 et $2160 \mu \mathrm{S} / \mathrm{cm}$ dans la CA de Thiès-Est et entre 685 à $1728 \mu \mathrm{S} / \mathrm{cm}$ dans la CA de Thiès-Ouest.

La situation est anormalement particulière à Dabaye $(2630 \mu \mathrm{S} / \mathrm{cm})$ et Diakhao base dans la CA Thiès-Nord ; et à Diamaguène dans CA Thiès à l'Est où la $\mathrm{CE}$ est supérieure $2000 \mu \mathrm{S} / \mathrm{cm}[2]$.

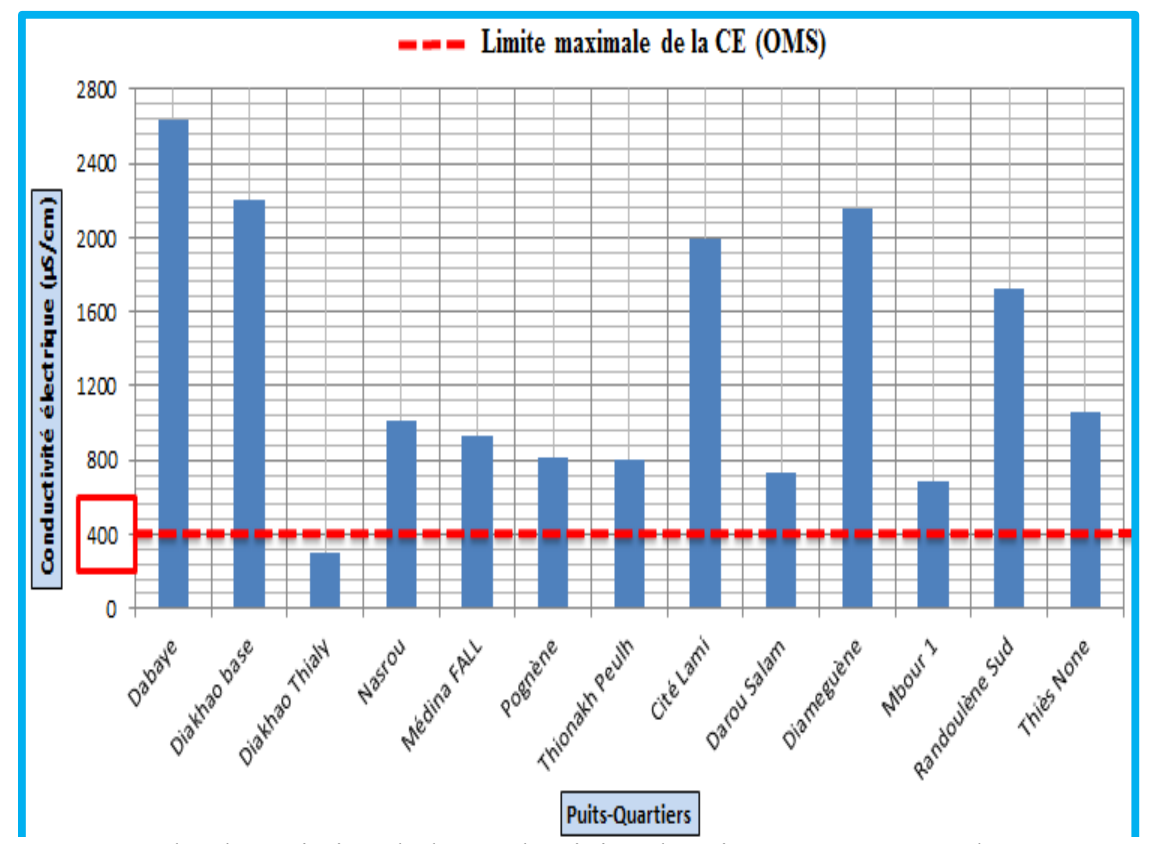

Figure 2:- Courbe de variation de la conductivité électrique par rapport à la norme OMS. 


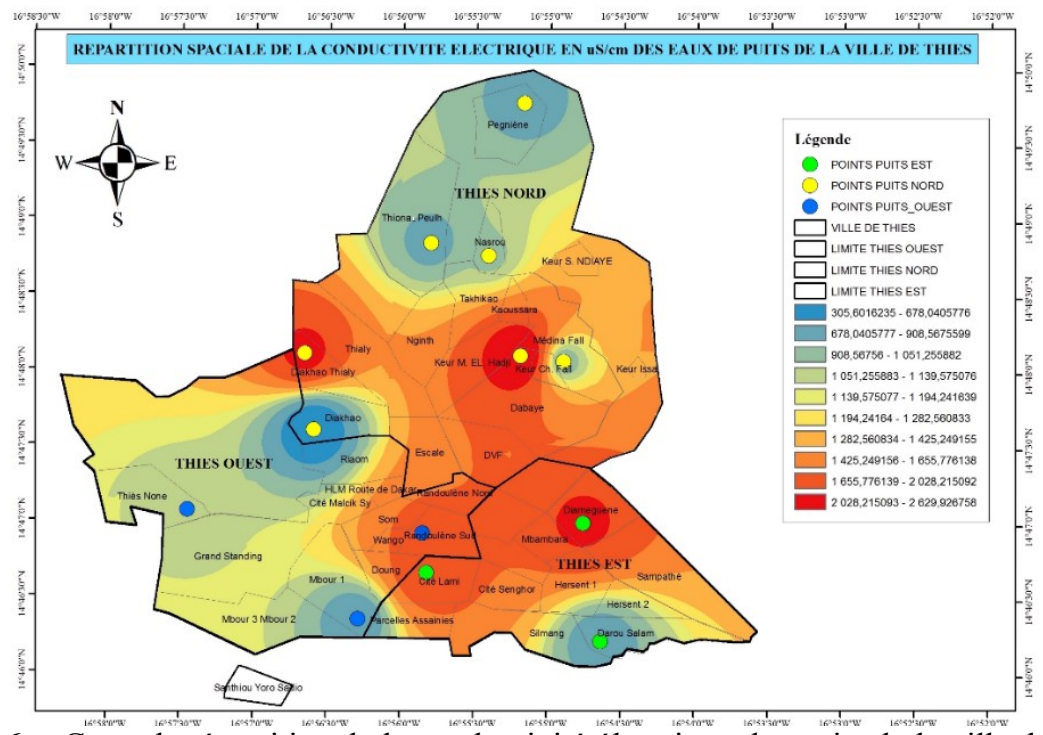

Figure 6 :- Carte de répartition de la conductivité électrique des puits de la ville de Thiès.

Tableau 1:- Variation de la minéralisation dans les 3 CA de Thiès.

\begin{tabular}{|l|c|l|}
\hline \multicolumn{1}{|c|}{ Puits/Quartier } & Conductivité électrique $\boldsymbol{\mu S / c m}$ & \multicolumn{1}{c|}{ Minéralisation globale } \\
\hline Dabaye & 2630 & Minéralisation élevée \\
\hline Diakhao base & 2200 & Minéralisation élevée \\
\hline Diakhao Thialy & 305 & Minéralisation moyenne \\
\hline Diouma Nasrou & 1010 & Minéralisation élevée \\
\hline Médina FALL & 934 & Minéralisation importante \\
\hline Pognène & 819 & Minéralisation importante \\
\hline Thiona Peulh & 798 & Minéralisation importante \\
\hline Cité Lami & 1994 & Minéralisation élevée \\
\hline Darou Salam & 730 & Minéralisation importante \\
\hline Diameguène & 2160 & Minéralisation élevée \\
\hline Mbour 1 & 685 & Minéralisation importante \\
\hline Randoulène Sud & 1728 & Minéralisation élevée \\
\hline Thiès None & 1060 & Minéralisation élevée \\
\hline
\end{tabular}

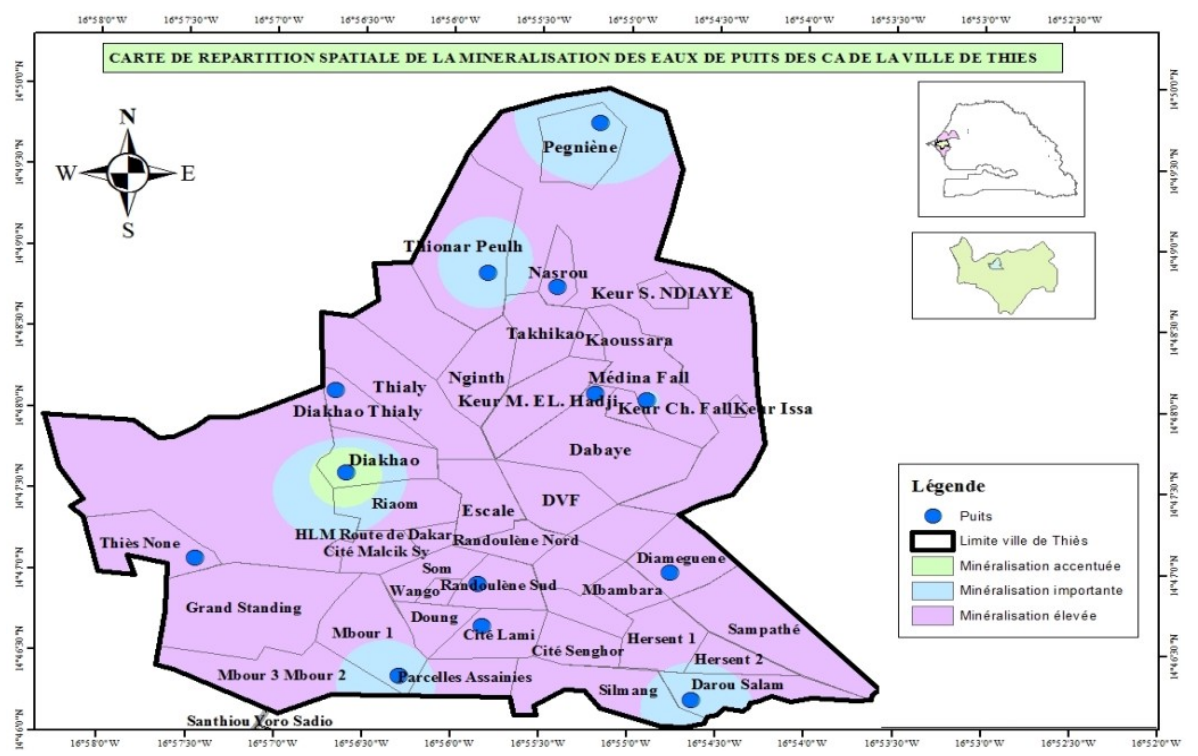

Figure3:- Carte de répartition de la minéralisation des eaux de puits de la ville de Thiès. 


\section{La dureté totale}

La dureté des eaux souterraines est fonction du milieu géologique où elles s'écoulent. La figure 8 ci-dessous montre l'occupation de valeurs élevées de dureté sur l'ensemble du territoire communal de la ville de Thiès, qui s'explique par la forte présence de formations calcaires dans son sous-sol. Ainsi, nous avons 7,69\% d'eau dure, 7,69 \% d'eau légèrement dure et $84,62 \%$ d'eau très dure dans la CA de Thiès-Nord; et $100 \%$ d'eaux très dures dans les CA de Thiès-Est et de Thiès-Ouest. Par ailleurs, une dureté élevée constitue un risque important d'entartrage des canalisations [6],[7].

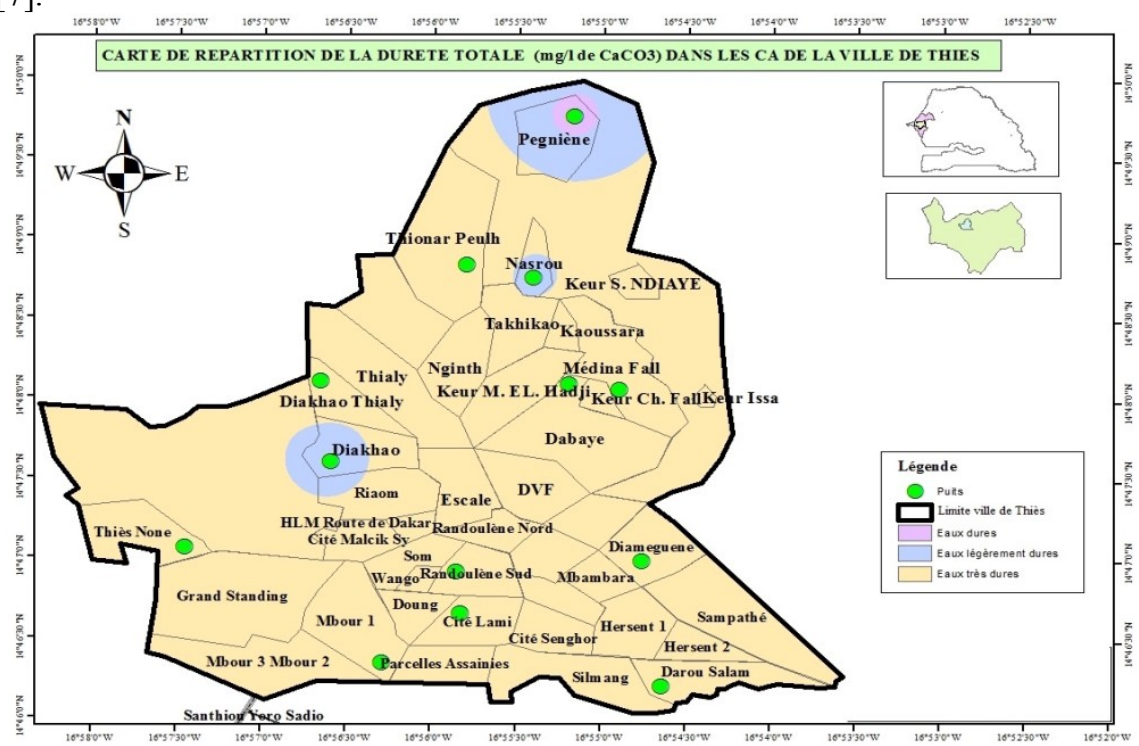

Figure 8:- Carte de répartition de la dureté totale des eaux de la ville de Thiès.

\section{Les Nitrates}

L'analyse des nitrates dans les eaux permet d'obtenir des informations sur la présence de matières organiques dans le sol [8]. Ces nitrates sont naturellement présents mais en faible quantité dans les sols. Ils constituent aujourd'hui le principal indicateur d'une dégradation des ressources en eau [6]. L'augmentation des teneurs constatées est notamment le résultat d'activités humaines.

Ainsi, la superposition des cartes piézométrique altimétriques et de la répartition de la teneur en nitrates dans les trois CA montre une nette coïncidence entre la zone de fortes teneurs en nitrates et la zone de basse altitude qui encaisse les matières organiques terrestres (décharges) drainées par les eaux de ruissellement pluviales d'une part, mais également le drainage imposé par la piézométrie de la nappe d'autre part.

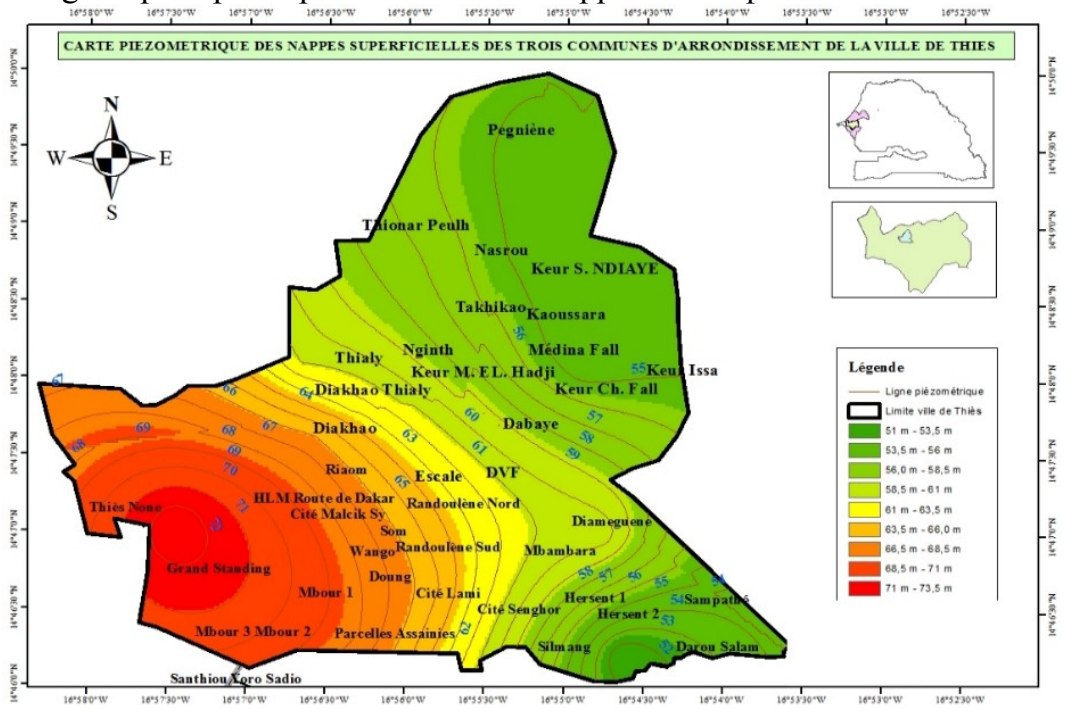

Figure 9:- Carte de répartition de la piézométrie dans les puits. 


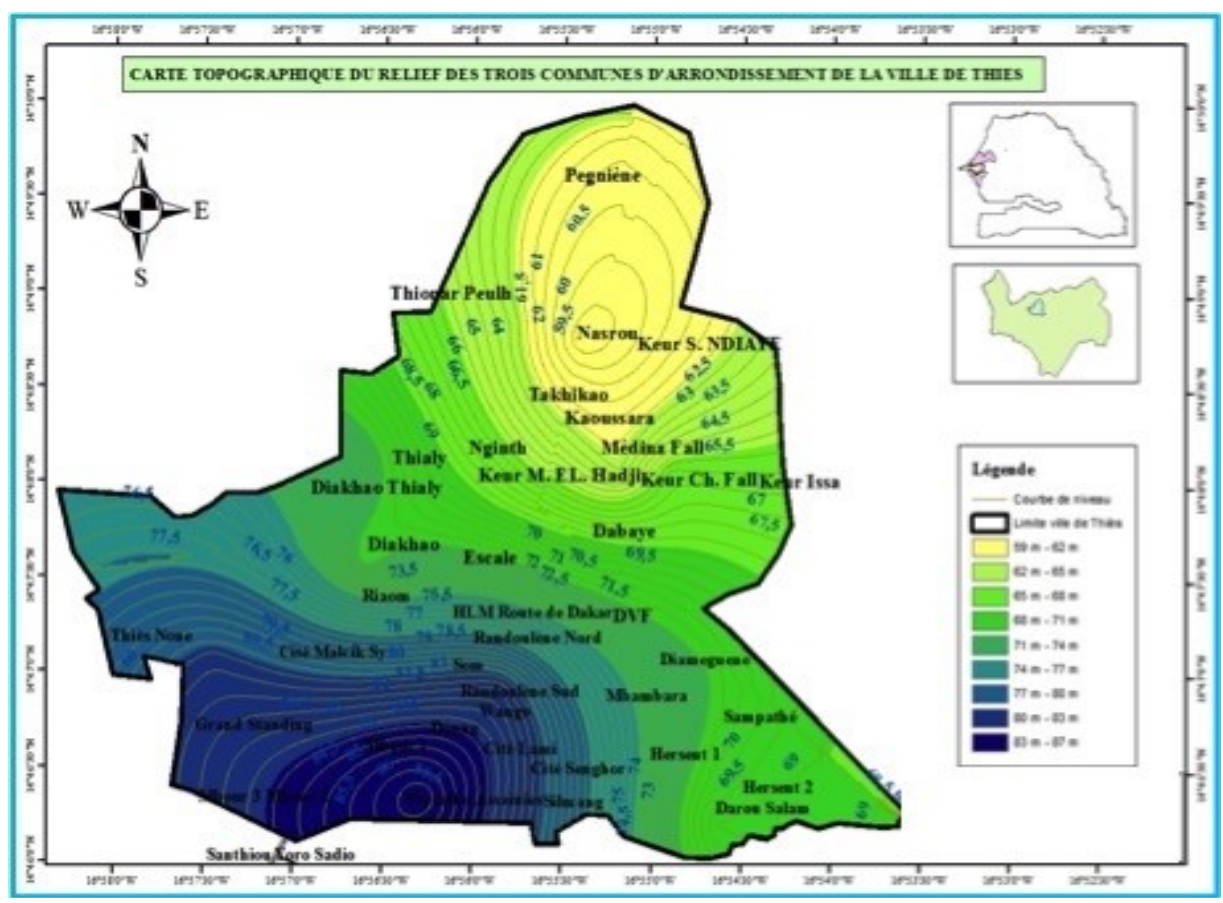

Figure 4:- Carte de répartition altimétrique.

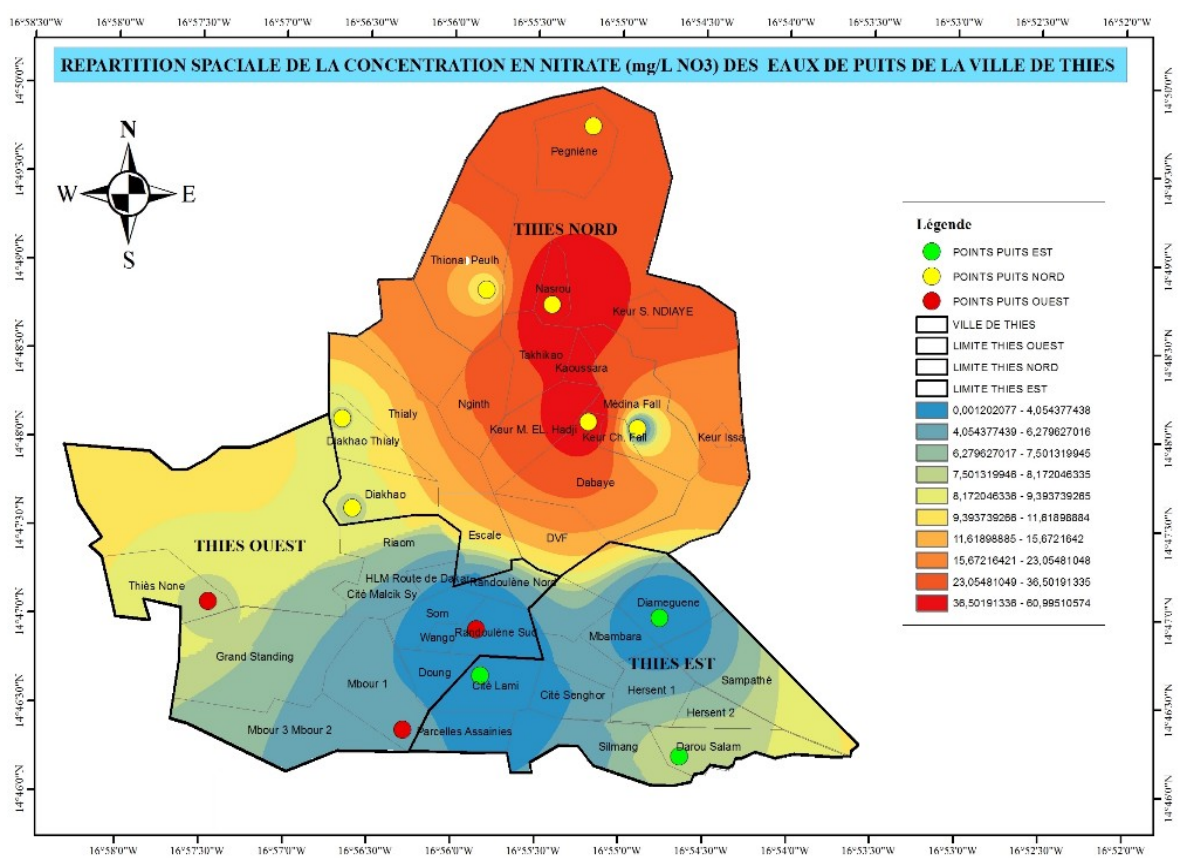

Figure 5:- Carte de répartition de la teneur en nitrates des puits de Thiès.

Les résultats d'analyses chimiques dans la ville de Thiès représentés à la figure 12 nous renseigne que les concentrations en nitrates évoluent entre $0,05 \mathrm{mg} / 1$ et $61 \mathrm{mg} / \mathrm{l}$. Les puits ayant les plus fortes concentrations en nitrates et qui dépassent la norme OMS (50 mg/l) sont ceux de Dabaye et de Nasrou, tous dans la CA de Thiès-Nord. Les plus faibles teneurs en nitrates sont enregistrées dans la CA de Thiès-Est. 


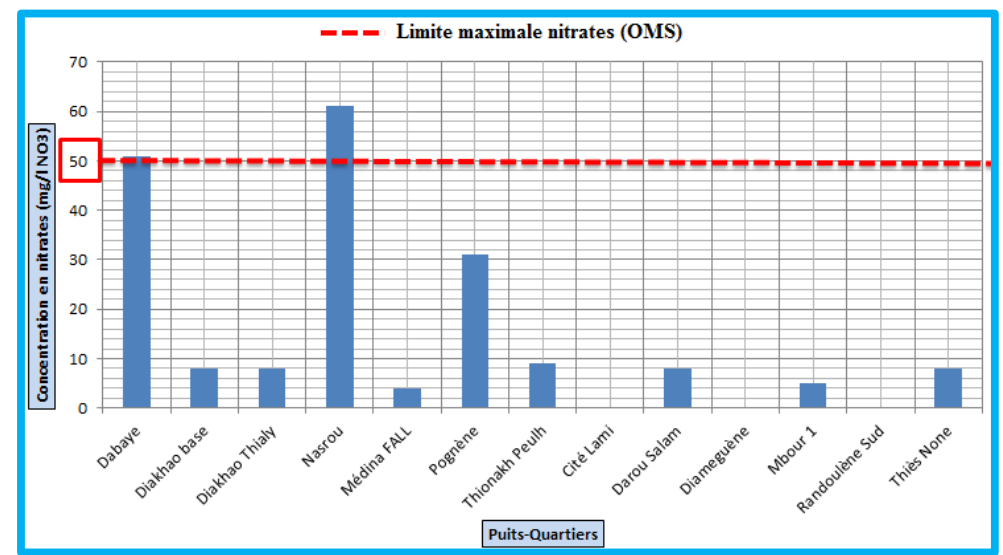

Figure 6:- Variation de la teneur en nitrates par rapport à la norme OMS.

Par conséquent la consommation de cette eau comme eau de boisson devrait être interdite aux femmes enceintes et aux nourrissons pour prévenir la méthémoglobinémie ou «Baby Blue Syndrome ", dans lequel le sang perd sa capacité à transporter l'oxygène en quantité suffisante [6].

\section{Les Nitrites}

Les eaux qui renferment des nitrites sont considérées comme suspectes, car souvent associée une détérioration de la qualité microbiologique [3], [9].

En effet, l'accroissement du taux de nitrates dans les eaux souterraines superficielles est dû pour une large part aux rejets d'eaux usées, pour la plupart issues des fosses septiques. Les nitrates en eux-mêmes ne présentent pas de danger particulier pour la santé, c'est leur transformation en nitrites dans l'estomac qui peut être toxique. Cette transformation se fait par le phénomène chimique de la réduction par l'élimination d'oxygène. Une trop forte concentration de nitrites dans l'organisme peut provoquer des maladies graves comme les cyanoses, en particulier chez les nourrissons dont l'alimentation est constituée essentiellement de lait réhydraté. Les teneurs en nitrites sont très variables et sont réparties telles que représentées à la figure 13.

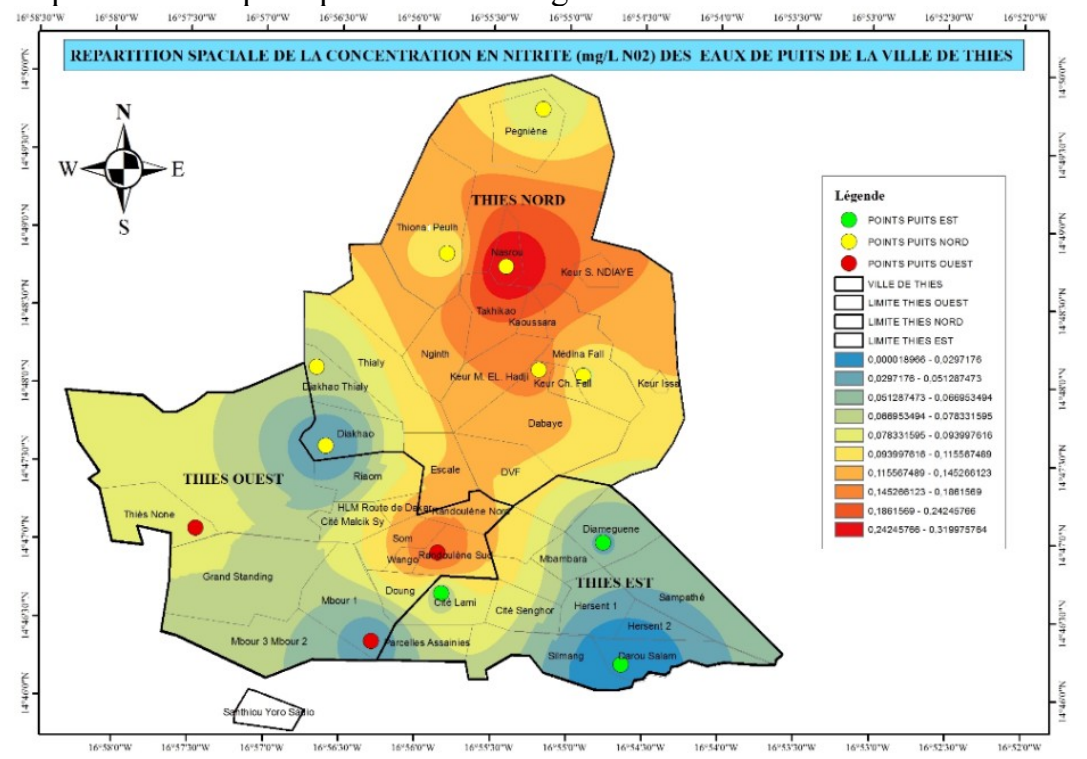

Figure 7:- Carte de répartition de la teneur en nitrite des puits de la ville de Thiès.

1. $0,03 \mathrm{mg} / 1 \mathrm{NO}_{2}{ }^{-}$à $0,32 \mathrm{mg} / 1 \mathrm{NO}_{2}^{-}$dans la $\mathrm{CA}$ de Thiès-Nord ;

2. $0 \mathrm{mg} / \mathrm{l} \mathrm{NO}{ }_{2}^{-}$à $0,07 \mathrm{mg} / 1 \mathrm{NO}_{2}^{-}$dans la CA de Thiès-Est et

3. $0,04 \mathrm{mg} / 1 \mathrm{NO}_{2}^{-}$à $0,19 \mathrm{mg} / 1 \mathrm{NO}_{2}^{-}$dans la $\mathrm{CA}$ de Thiès-Ouest. 
La figure 14 nous montre que la presque totalité des puits respecte la norme OMS de teneur en nitrites, à l'exception de celui de Nasrou, situé dans la CA de Thiès-Nord.

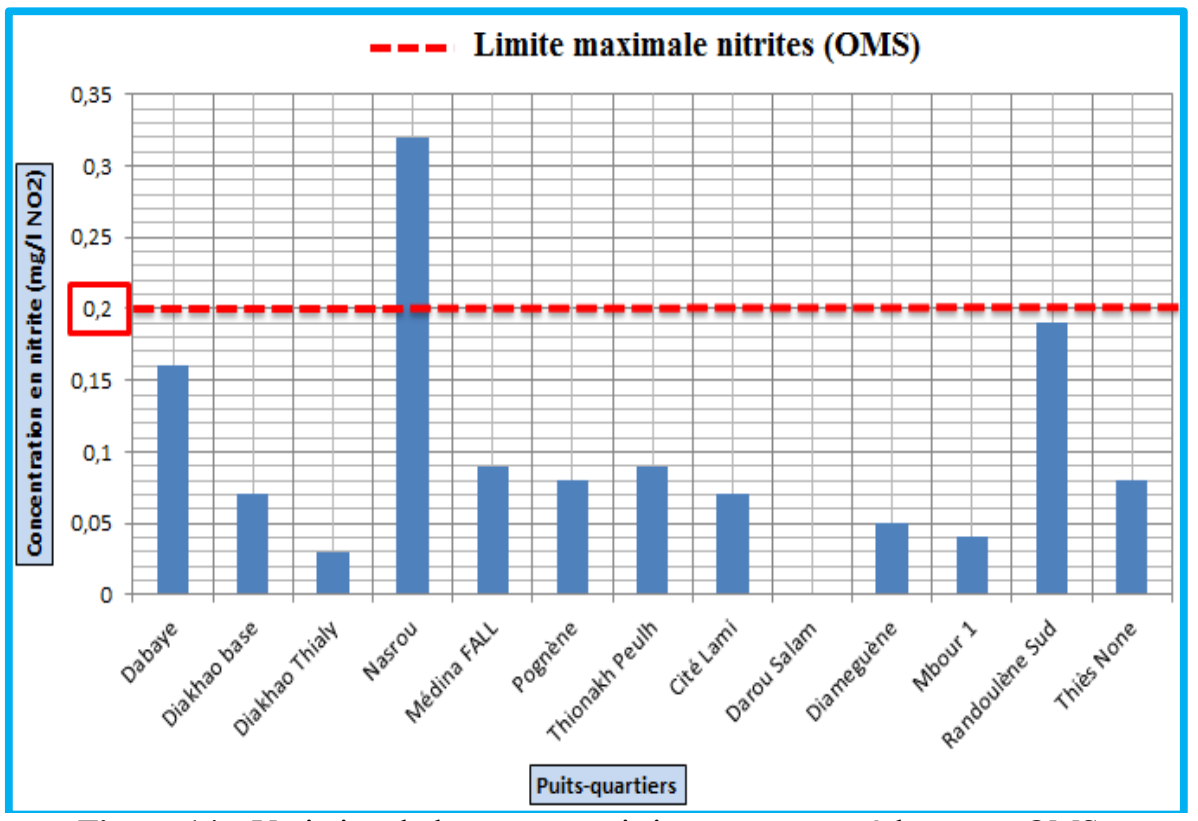

Figure 14:- Variation de la teneur en nitrites par rapport à la norme OMS.

\section{Les Phosphates}

Le phosphore, symbolisé par la lettre $\mathrm{P}$ est largement répandu dans la nature. Il est généralement lié à de l'oxygène sous forme de phosphate (PO4) [9].

Les concentrations en phosphates dans les 13 puits échantillonnés dans la ville de Thiès varient entre $0,94 \mathrm{mg} / 1$ $\mathrm{PO}_{4}{ }^{3-}$ à $39 \mathrm{mg} / \mathrm{PO}_{4}{ }^{3-}$. La figure 15 montre la répartition des concentrations en phosphates dans les $3 \mathrm{CA}$ de la ville de Thiès.

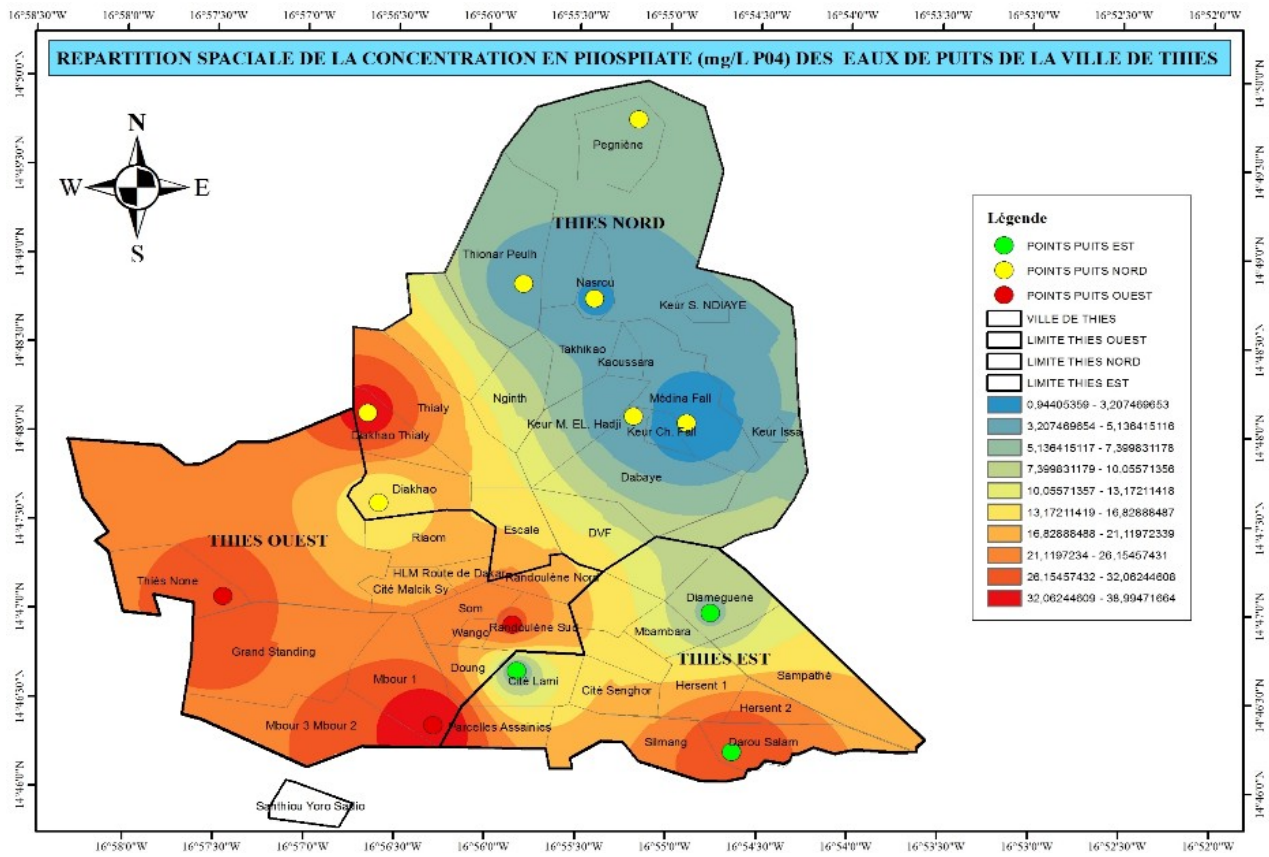

Figure 8:- Carte de répartition de la teneur en phosphates dans les puits de la ville de Thiès. 
1. dans la CA de Thiès-Nord : la plus faible concentration en phosphates dans les eaux de puits est enregistrée à Médina-FALL $\left(0,94 \mathrm{mg} / 1 \mathrm{PO}_{4}^{3-}\right)$, et la plus élevée à Diakhao-base avec $35 \mathrm{mg} / 1 \mathrm{PO}_{4}{ }^{3-}$;

2. dans la CA de Thiès-Est : l'ouvrage de Cité Lami a la plus faible concentration avec $5 \mathrm{mg} / \mathrm{PO}_{4}{ }^{3-}$, tandis que celui de Darou-Salam avec $31 \mathrm{mg} / 1 \mathrm{PO}_{4}{ }^{3-}$ est la plus grande.

3. dans la CA de Thiès-Ouest : la plus faible concentration en phosphates est notée au puits de Randoulène-Sud avec $28 \mathrm{mg} / \mathrm{PO}_{4}{ }^{3-}$ là où celui de Mbour 1 donne la valeur maximale avec $39 \mathrm{mg} / 1 \mathrm{PO}_{4}{ }^{3-}$.

Cependant, au regard des figures 15 et 16, seuls les ouvrages de captage de Dabaye, Nasrou, Médina-FALL et Thionakh se trouvant dans la CA de Thiès-Nord respectent la norme de l'OMS $\left(5 \mathrm{mg} / 1 \mathrm{P}_{2} \mathrm{O}_{5}\right)$. Tous les autres puits situés dans les CA de Thiès-Est et Thiès-Ouest ont des concentrations en phosphates qui dépassent largement la norme de teneur en phosphates.

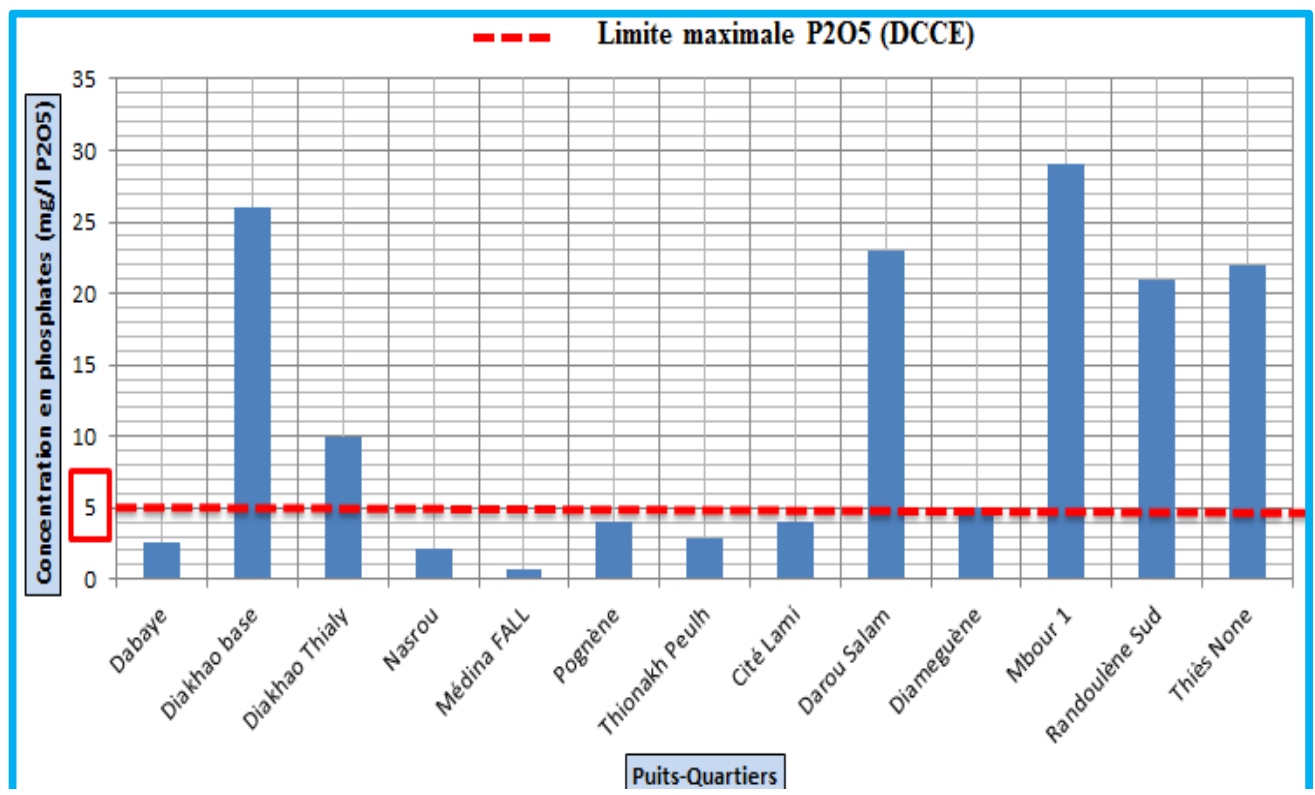

Figure 9:- Variation de la teneur en phosphates par rapport à la norme OMS.

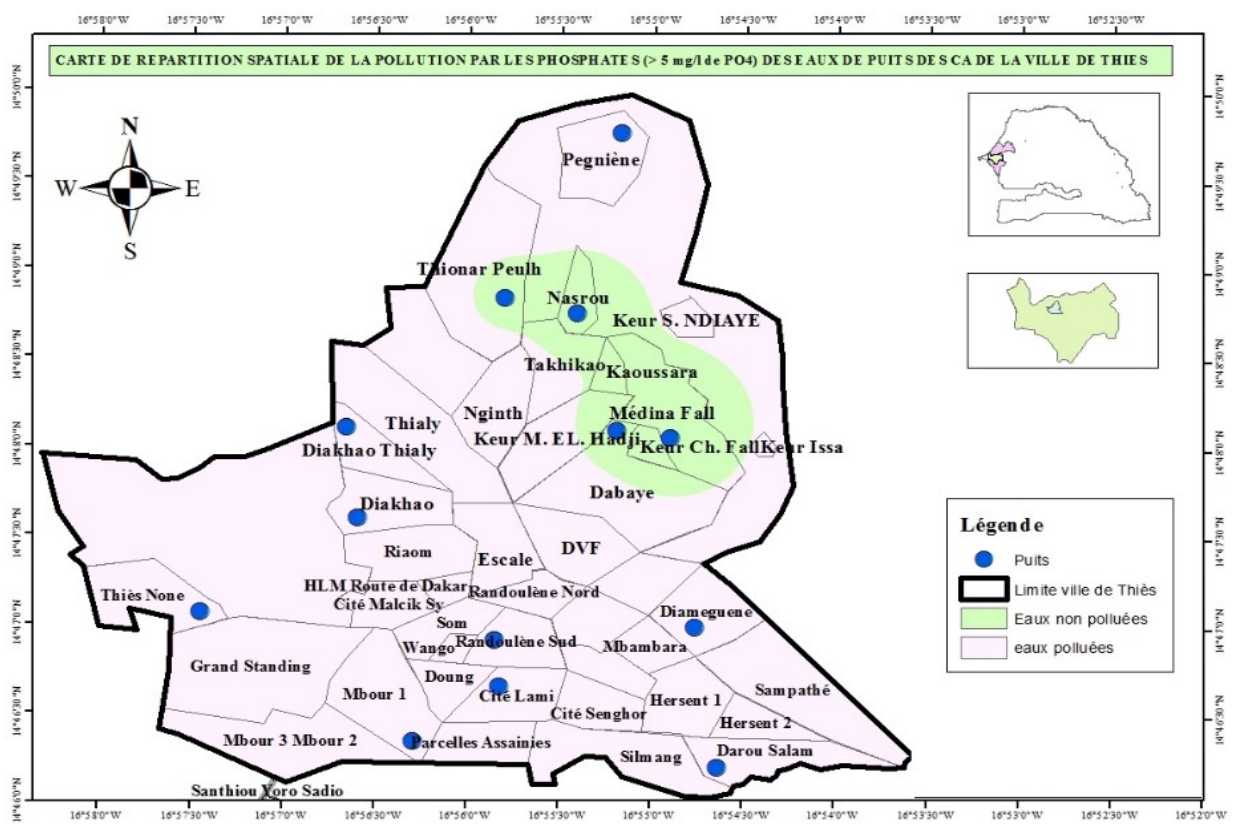

Figure 10:- Carte de répartition de la pollution en phosphates dans les puits de la ville de Thiès. 
Ces fortes concentrations en phosphates dans les CA de Thiès-Est et Thiès-Ouest, dénotent du faible taux d'accès à l'assainissement collectif dans ces zones d'une part, et la précarité des fosses septiques d'autre part à proximité des puits qui ont été étudiés.

L'éloignement de toutes autres sources potentielles de pollution telles que les purins et les dépotoirs d'ordures, nous poussent à en tirer que ces fosses septiques constituent la source principale de pollution par les phénomènes d'infiltration des polluants dans les nappes superficielles des trois CA de Thiès. Ainsi nous pouvons dire que toutes les eaux des puits sont polluées en phosphates si on se base sur les teneurs observées.

Dès lors, les teneurs en phosphates supérieures au seuil de pollution de $0,5 \mathrm{mg} / \mathrm{l}$ de P04 notées au niveau de tous les puits constituent un fort indice de pollution.

\section{L'analyse Des Facies Chimiques}

L'analyse du faciès chimique est effectuée à l'aide des diagrammes de Pipper et de Schoëller-Berkaloff. Le diagramme de Piper représenté à la figure 18 est une représentation graphique de la chimie d'échantillons d'eau [10].

Celui de Schoëller-Berkaloff représenté à la figure 19 constitue la représentation graphique du faciès chimiques d'échantillons d'eau [3], [11].

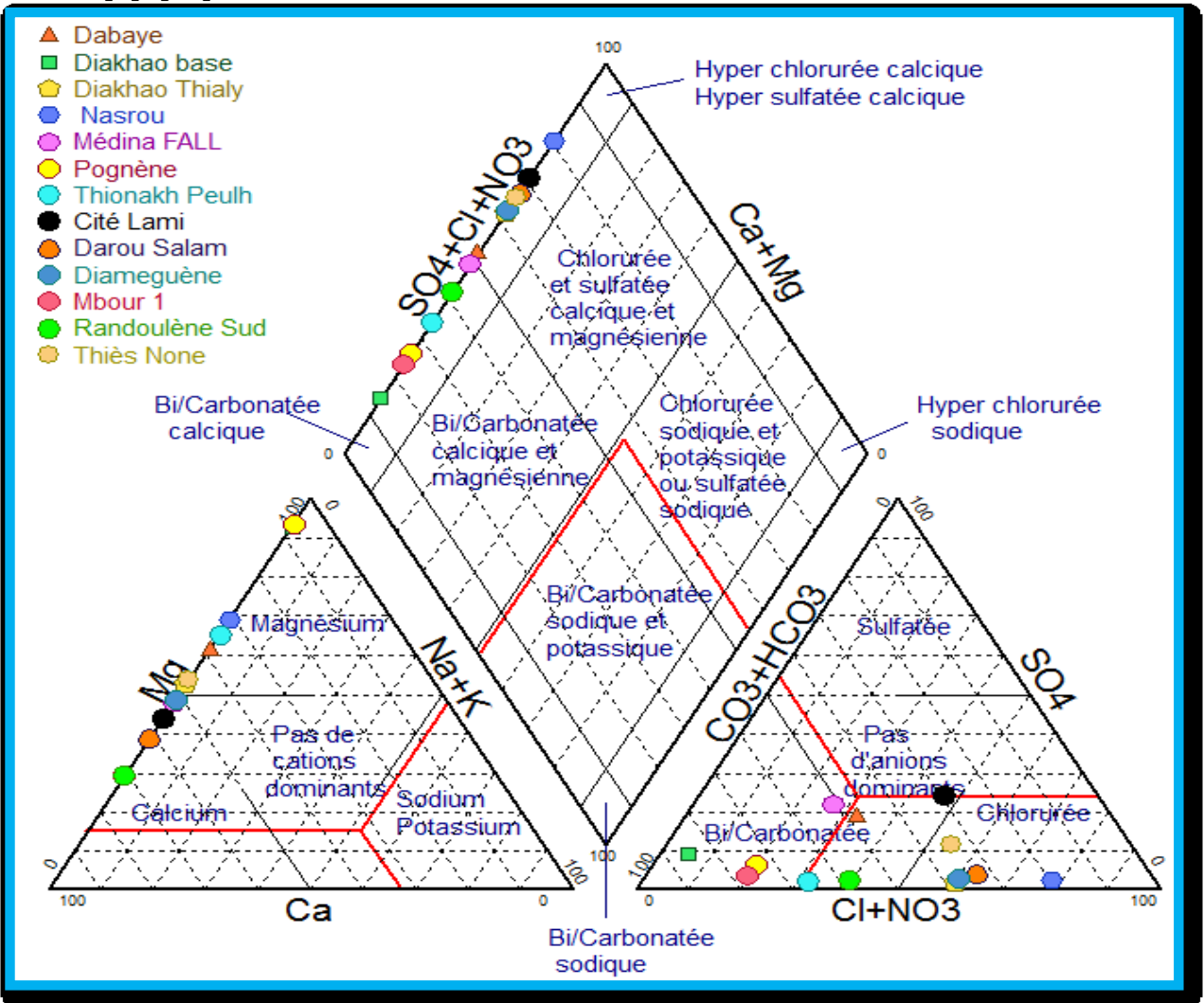

Figure 18:- Diagramme de Pipper. 


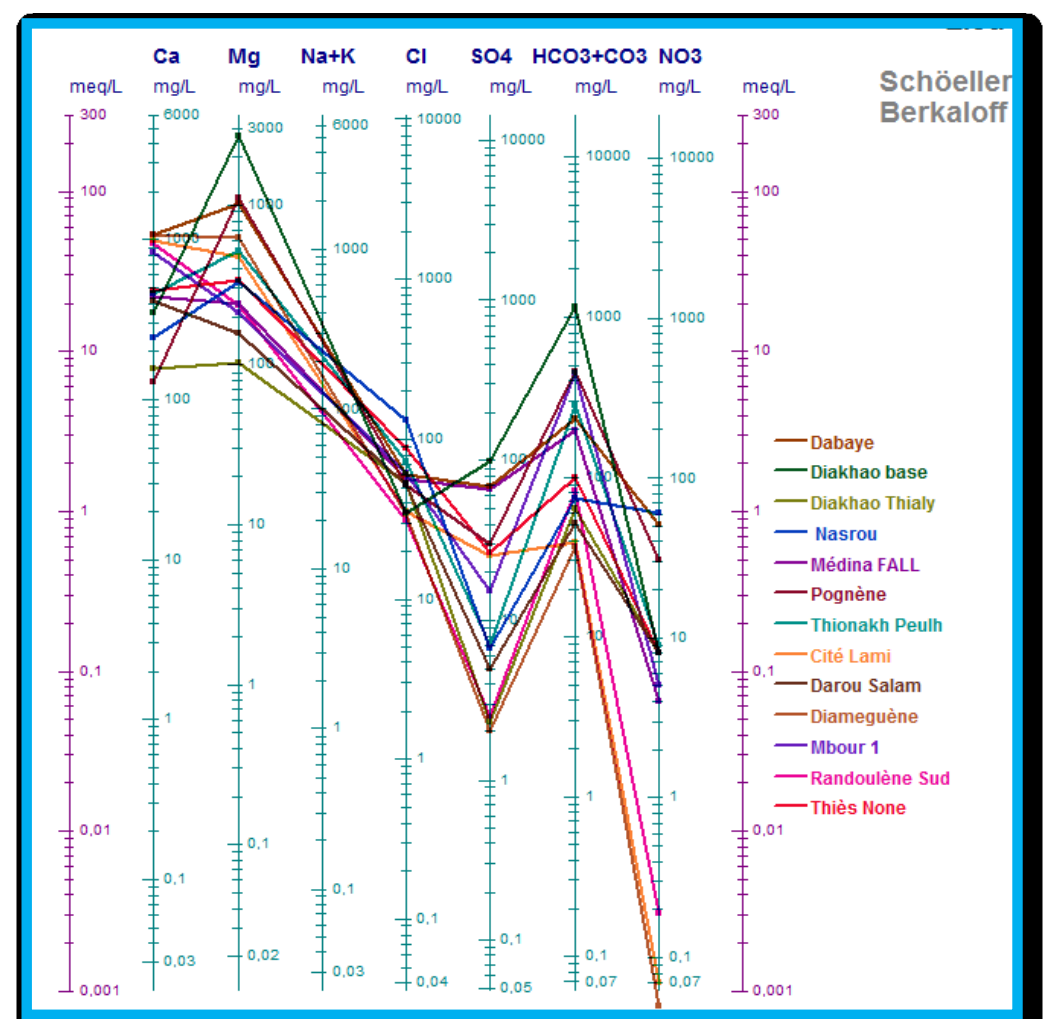

Figure 11:- Diagramme de Schoëller-Berkaloff.

L'analyse de ces deux diagrammes renseigne que les eaux de la nappe superficielle de la ville de Thiès présentent deux faciès chimiques :

1. Un faciès Chloruré sulfaté et calcique magnésique constaté à Nasrou, Cité Lami, Darou Salam, Thiès None, Diamaguène, Diakhao Thialy, Médina Fall et Dabaye ;

2. Un faciès Bicarbonaté calcique et magnésique mis en évidenceà Mbour 1, Thionakh Peulh, Randoulène Sud, Diakhao Base et Pognène.

\section{Analyses Microbiologiques : Les Coliformes Fecaux}

L'examen microbiologique de l'eau a pour objet fournir des informations quant à la potabilité relative au risque d'ingestion de micro-organismes qui causent des maladies, provenant généralement d'une contamination par des matières fécales humaines ou d'autres animaux à sang chaud [12].

Les coliformes fécaux font partie des sous-groupes de bactéries coliformes qui fermentent le lactose à $44,5 \pm 0,2^{\circ} \mathrm{C}$ sous 24 heures [13].

L'espèce la plus fréquemment associée à ce groupe bactérien est l'Escherichia coli (E. coli) et, dans une moindre mesure, certaines espèces des genres Citrobacter, Enterobacter et Klebsiella). La bactérie E. coli représente toutefois 80 à $90 \%$ des coliformes thermo-tolérants détectés. La présence de coliformes fécaux témoigne habituellement d'une contamination d'origine fécale qui peut amener diverses maladies [14]; dont la plus fréquente est la gastro-entérite. Habituellement sans gravité, mais toutefois, elle peut avoir de graves conséquences sur la santé pour les enfants, les personnes âgées et les personnes dont le système immunitaire est affaibli [13].

La présence des animaux à sang chaud (troupeaux de bœufs) aux environs de puits n'a été rencontrée qu'à Thionakh Peulh, avec une marre de boue de bouse de vaches autour du puits, malgré une présence de coliformes fécaux dans les eaux de puits (moins de 1 micro-organisme par $100 \mathrm{ml}$ ).

La présence d'argile marneuse dans les premiers horizons du sol peut empêcher les polluants de progresser dans cette zone jusqu'à la nappe, telle qu'illustrée à la figure 20 ci-dessous. 


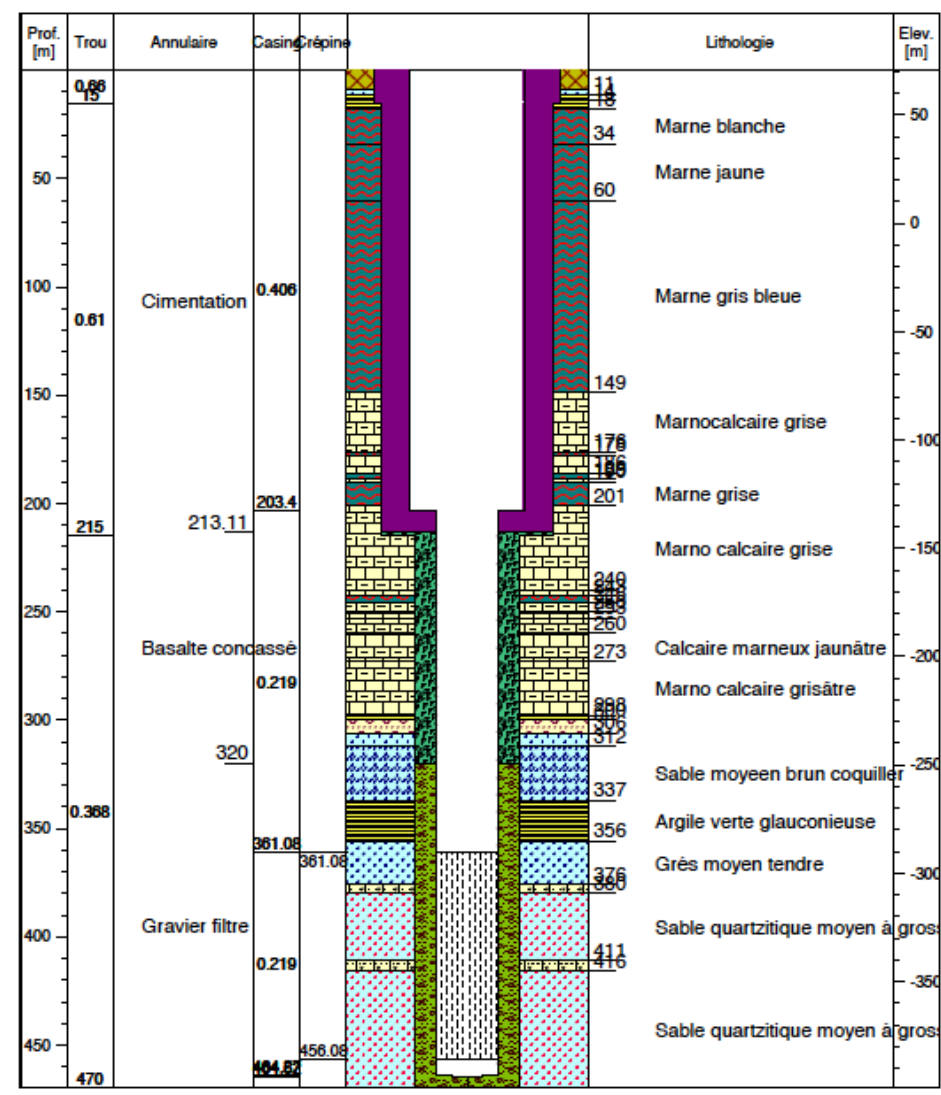

Figure 12:- Coupe lithologique du forage F10 situé à Thionakh Peulh.

Nous pouvons dire de façon générale que la présence de coliformes fécaux dans les eaux de puits étudiées dépasse partout la limite de l'OMS ( $<0$ UFC/100). Cette pollution est généralement d'origine anthropique bien vrai que les animaux à sang chaud aussi causent les mêmes effets. Mais d'après les enquêtes sur le terrain, l'évaluation de l'impact des fosses septiques dans l'environnement des puits montre que les fosses septiques concentrées aux alentours des puits sont les principales sources de pollution.

Les résultats d'analyses microbiologiques des eaux de puits de la ville de Thiès représentés dans le tableau 2 révèlent que la totalité des ouvrages est polluée par les coliformes fécaux, selon la norme de référence NS 05-044 des méthodes d'analyses utilisées.

Tableau 2:- Résultats des analyses microbiologiques des eaux de puits de la ville Thiès.

\begin{tabular}{|c|c|c|c|}
\hline Numéro d'ordre & Échantillon & Localité & Résultats \\
\hline 1 & Échantillon $\mathrm{N}^{\circ} 1$ & Darou-Salam & - de 1 micro-organisme par $100 \mathrm{~mL}$ \\
\hline 2 & Échantillon $\mathrm{N}^{\circ} 2$ & Dabaye & $400 \mathrm{UFC} / \mathrm{mL}$ \\
\hline 3 & Échantillon $\mathrm{N}^{\circ} 3$ & Médina-FALL & $4400 \mathrm{UFC} / \mathrm{mL}$ \\
\hline 4 & Échantillon $\mathrm{N}^{\circ} 4$ & Nasrou & $300 \mathrm{UFC} / \mathrm{mL}$ \\
\hline 5 & Échantillon $\mathrm{N}^{\circ} 6$ & Pognène & $400 \mathrm{UFC} / \mathrm{mL}$ \\
\hline 6 & Échantillon $\mathrm{N}^{\circ} 7$ & Thionakh Peulh & - de 1 micro-organisme par $100 \mathrm{~mL}$ \\
\hline 7 & Échantillon $\mathrm{N}^{\circ} 12$ & Mbour I & - de 1 micro-organisme par $100 \mathrm{~mL}$ \\
\hline 8 & Échantillon $\mathrm{N}^{\circ} 13$ & Thiès None & $250 \mathrm{UFC} / \mathrm{mL}$ \\
\hline
\end{tabular}

La concentration en coliformes fécaux (Escherichia Coli) recherchés dans les eaux de puits de la ville de Thiès varie de moins d'un micro-organisme par ml à 4400 UFC/100 ml, dépassant largement les normes de l'OMS. La figure 21 ci-dessous montre la répartition des concentrations en micro-organismes (Coliformes fécaux) dans les différents ouvrages des trois (03) CA de la ville de Thiès. 


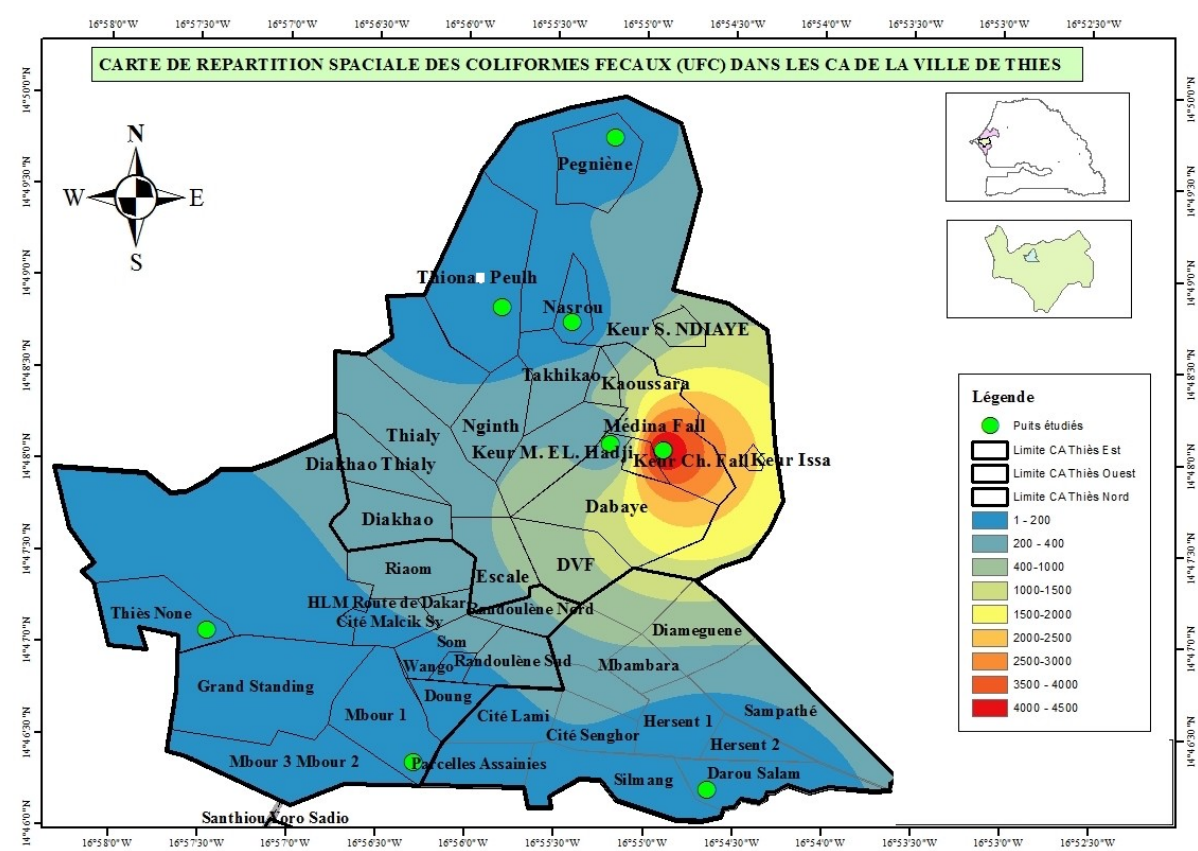

Figure13:- Carte de répartition de la pollution microbiologique par les Coliformes fécaux.

La figure 21 nous montre que la répartition varie de :

1. moins de 1 micro-organisme par $\mathrm{ml}$ à $4400 \mathrm{UFC} / 100 \mathrm{ml}$ dans les puits de Thiès-Nord ;

2. moins de 1 micro-organisme par $\mathrm{ml}$ à $250 \mathrm{UFC} / 100 \mathrm{ml}$ dans les puits de Thiès-Ouest ;

3. et tourne autour de moins d'l micro-organisme par ml dans le puits de la CA de Thiès-Est.

Cette pollution par les coliformes fécaux est d'origine domestique notamment dans le cas d'un assainissement collectif ou individuel défectueux, des substances indésirables contenues dans les eaux vannes et les eaux ménagères peuvent être transférées à la nappe (matières organiques, détergentes, solvants, antibiotiques, micro-organismes.)

Les eaux souterraines des puits peu profonds sont contaminées par les déchets humains et animaux par le biais de ces polluants sous forme de chlorures, des phosphates et des nitrates. Ces dernières sont très solubles dans l'eau et constituent de nos jours la principale cause de la pollution des eaux souterraines [1]. Les pollutions microbiologiques se rencontrent surtout dans les aquifères à perméabilité de fissure (craie, massifs calcaires), dans lesquels la fonction épuratrice du sous-sol ne peut s'exercer et dans lesquels la matière organique est dégradée partiellement. Ces émergences de type karstique avec des circulations souterraines rapides sont par conséquence très vulnérables à cette pollution.

C'est le cas de la presque totalité des acquières captés par les puits échantillonnés dans les trois CA de la ville de Thiès, qui sont de nature gravelo-marno-calcaires sur toute la couche sus-jacente, donc favorables à la circulation des polluants d'origines fécales, d'où la forte présence de coliformes fécaux qui confirme l'impact réel des fosses septiques qui ne respectent aucune norme d'implantation et de construction, sur les eaux des nappes phréatiques captées par ces puits.

\section{Conclusion:-}

L'utilisation de différentes méthodes de classifications des eaux des puits des trois (03) communes d'arrondissement de Thiès telles que les classifications de Schoeller-Berkaloff et Piper montrent l'existence d'un faciès chimique dominant qui est le faciès de nature chloruré-sulfaté et calcique-magnésique. Ceci est une confirmation de la pollution des eaux des puits par les sulfates et les chlorures.

Ainsi, au regard des résultats d'analyses physicochimiques et microbiologiques, la forte présence de Coliformes fécaux dans les eaux des puits échantillonnés nous constatons un impact réel des ouvrages d'assainissement que sont les fosses septiques, généralement utilisés dans notre zone d'étude, sur la qualité de l'eau des nappes. 
A Thionakh Peulh, Darou Salam et Mbour1 les faibles teneurs en coliformes fécaux (moins de 1 micro-organisme par $\mathrm{ml}$ ) enregistrés, s'expliquent par la présence de couche marno-argileuse peu perméable sus-jacente à la couche aquifère et qui rend difficile transport des polluants microbiologiques vers la nappe. Par contre, la presque totalité des aquifères captés par les puits sont de nature graveleux-marno-calcaires, donc favorables à la circulation des polluants d'origines fécales, d'où la forte présence de coliformes fécaux qui confirme l'impact des fosses septiques qui ne respectent aucune norme d'implantation encore moins de construction.

\section{Références:-}

1. MinaA., A. Saadia, S. B. Abdes et H. Ben Younes, 2007. Cartographie de la vulnérabilité à la pollution des eaux souterraines: Application à la plaine du Gharb,Revue des sciences de l'eau de l'Université du QuébecINRS-Eau, Terre et Environnement vol. 20, n12, pp. 185-199.

2. O. M. S. (OMS), 2017. Directives de qualité pour l'eau de boisson (4e édition)ISBN 978-92-4-254995-9.

3. Rodier Jean, L. Bernard, M. Nicole et coll., 2009. L'Analyse de l'eau, Paris: Dunod ISBN 978-2-10-054179-9.

4. C. Joseph, C. Rodier, M. Soulie, F. Sinegre and R. Baylet., 1998. Approche des transferts de pollution bactérienne dans une crue karstique par l'étude des paramètres physico-chimique. Revue des sciences de l'eau Université du Québec - INRS-Eau, Terre et Environnement, Vol. 1, pp. 73-106.

5. Mouvet, L. Chéry et C., 2000. Principaux processus physico-chimiques et biologiques intervenant dans l'infiltration des produits polluants et leur transfert vers les eaux souterraines. 8, s.l. : La Houille Blanche, Vol. 7, pp. 82-88.

6. M. Ali BEN et C. et. E. H. R. bdelkader, 2011. Qualité de la source Ain Hamma et effet du déversement de ses eaux sur la qualité physico-chimique et bactériologique des eaux de l'Oued Khoumane, Maroc, Afrique Science, vol. $7, \mathrm{n}^{\circ} 12$, pp. $115-130$.

7. Jeannin, P.-Y. and Sauter, M., 1998. Analysis of karst hydrodynamic behavior using global approaches: A review. Bulletin d'Hydrogéologie de l'Université de Neuchâtel, 16: 31-48.

8. Degbey C., Makoutode M., Ouendo E.M., De BrouwerC., 2010. Pollution physico-chimique et microbiologique de l'eau des puits dans la Commune d'Abomey-Calavi au Bénin. 6, s.l. : International Journal of Biological and Chemical Sciences, Vol. 4, n¹6, 2010.

9. LAMRIBAH A., M. BENAJIBA, Y. SAOUD, M. AHRIKAT et M. BENZAKOUR, 2013. Impact de la pollution urbaine sur la contamination par les nitrates et le nitrites de la nappe phréatique de Martil, Larhyss Journal. ISSN 1112-3680 n 114 .

10. Layachi G., G. Omar, B. Abderahmane, L. Mohamed et M. Claude, 2012. Evaluation de la salinité des eaux souterraines utilisées en irrigation et risques de dégradation des sols : exemple de la plaine de Meskiana (NordEst Algérien), vol. 6, pp. 141-160, 2012.

11. Hammoumi N El, S. M, L. B et M. L El, 2012. Évaluation de la qualité des eaux souterraines pour l'utilisation dans l'eau potable et l'agriculture : plaine de Tadla, Maroc,» vol. 8, n¹1, 2012.

12. Nola. M., N. T., K. N., S. Z. Togouet, S. P., M. M., B. A. M. Cl. et M. S. Foto, 2006. Transfert des bactéries fécales vers une nappe phréatique à travers une colonne de sol en région équatoriale : Revues des sciences de l'eau , 2006, Vol. 19, pp. 101-112.

13. Joret C., Volk et J. C., 1994. Paramètres prédictifs de l'apparition des coliformes dans les réseaux de distribution d'eau d'alimentation, Revue des sciences de l'eau, 1994, Vol. 7, pp. 131-152.

14. Hamid B. S., N. Nadine, S. Antione G. El, D. Rosette, M. Samir et O. Naim, 2007. Suivi de la qualité bactériologique des eaux de surface (rivière Nahr Ibrahim, Liban),» vol. 20, n¹2, 2007. 